\title{
Recent Advances in Body Area Network Technology and Applications
}

\author{
Matti Hämäläinen ${ }^{1}$ Xinrong $\mathbf{L i}^{2}$
}

Published online: 14 March 2017

(c) Springer Science+Business Media New York 2017

The use of wireless body area networks (WBAN) has been seen as a modern way to monitor human's health related parameters remotely and seamlessly. The WBAN is utilizing energy efficient sensor nodes distributed around the human body and a coordinator node that controls the personal network's operation. WBANs are utilizing shortrange radio technologies to convey vital sign information from the source to the final destination; either being electric health record or just a central hub, which can keep track and analyze the measured parameters on-site.

The reasoning adopted by the health service providers is to decrease the caregivers' workload, allowing freedom and mobility for the patients or other persons who need to be monitored. Finally, all these are targeting to improve patients' quality of life but also to decrease the healthcare costs.

Currently technologies, such as the dominant Bluetooth and especially Bluetooth Low Energy, but also ZigBee, IEEE802.15.6, etc. are available for commercial WBAN implementation. In Europe, the European Telecommunication Standard Institute (ETSI) is also developing its own standard for smart body area networks under the Technical Committee SmartBAN. Despite of the existing standards suitable for WBAN use, the research is still widely ongoing. And the prognoses are expecting even wider deployment of

Matti Hämäläinen

matti.hamalainen@oulu.fi

Xinrong Li

xinrong@unt.edu

1 Centre for Wireless Communications, University of Oulu, Oulu, Finland

2 University of North Texas, Denton, TX, USA wearable technology in healthcare and welfare related applications than it is realized so far.

However, WBAN is only one part of the whole concept forming an effective and dependable chain to deliver health related information. The medical information and communication technology (ICT) concept as a whole consists of backbone systems, data collection and analysis, safety and secrecy related issues, various kinds of detectors, energy efficient electronics, and so on. In addition, the data transmission path is typically heterogeneous, which means that information is passing through various radio and wired connections. Moreover, different medical instruments can produce incompatible information, which need to be collected, merged and analyzed jointly with other related information.

As can be seen, the medical ICT is multidiscipline research area, which involves communication, electronics, data and signal processing, medical etc. disciplines together. In this Special Issue, we are dealing with the diverse set of topics under the theme of personalized healthcare. The papers included in this Special Issue are extended versions of the papers originally presented in the 10th International Symposium on Medical Information and Communication Technology (ISMICT) in 2016 at Worcester Polytechnic Institute, Worcester, MA, USA. Seven papers are selected for this Issue.

The first paper "Theoretical and Experimental Approach for the Design of an Optical Wireless Physical Activity Monitoring System" by Clément Le Bas et al. investigates theoretically the optical wireless activity monitoring system's performance in terms of packet failure in a specific environment. Paper studies, in particular, the impact of the optical source directivity, the emitted optical power, the position of the motion sensor device on the body, and the number of optical receivers fixed on the room ceiling. 
Authors compare theoretical results with the experimental measurements for several configurations using a custommade wearable communicating device and concluded that optical measurement system can be used in physical activity monitoring indoors.

"Evaluation of ground loop through the floor in human body communication" by Ken Sasaki et al. addresses the contribution of the ground loop through the floor in human body communication (HBC) application via experimental research. In the study, a received signal strength was measured in two scenarios; two subjects were shaking hands and a subject was touching an off-body receiver placed on a stand. The subjects each wore a transmitter or a receiver on their wrist. In the study, different floor material were used. The subjects stood on a carpet covered metal floor, concrete floor, hardwood floor, and a wooden chair to be above the floor.

"Challenges in Real-Time Vital Signs Monitoring for Persons during Exercises" by Shinsuke Hara et al. discusses on the use of wearable technology in the everyday use by Japanese school children to monitor their vital signs in learning and physical training purposes. In addition, authors presented some technical problems they found, and introduced solutions to solve the existing problems.

"On-line EEG Denoising and Cleaning Using Correlated Sparse Signal Recovery and Active Learning" by Manish Gupta et al. introduces two new methods to identify and remove artefacts caused by mobility in electroencephalography (EEG) recordings. For example, artefacts arose by the eye movement can be removed from the EEG data using the algorithm presented in the paper. According to the authors, their algorithm can be applied to remove artefacts, e.g., from magnetoencephalography (MEG) data.

"Impact of IEEE 802.15.4 communication settings on performance in asynchronous two way UWB ranging" by Konstantin Mikhaylov et al. reports the results of an extensive experimental study revealing the effect of various communication settings on the accuracy of indoor human or machine localization using novel asymmetric localization protocol for ultra wideband signaling. Authors reported that the self-heating increasing the UWB transceiver's temperature has an impact on the localization accuracy.

"Modeling the Effect of Human Body on ToA Ranging Using Ray Theory" by Fardad Askarzadeh et al. proposes an analytical Uniform Geometrical Theory of Diffraction (UTD) approach, based on a hybrid applicability of conductor cylinder, wedge, and screen models, to analyze behavior of the first path in proximity of human body that is in angular motion. In the paper, the authors also validate the proposed analytical method with ultra wideband measurements.

"Evaluation of LoRa LPWAN Technology for Indoor Remote Health and Wellbeing Monitoring" by Juha Petäjäjärvi et al. studies the performance of low power wide area network (LPWAN) technology indoors. Even though targeted for outdoor applications, LoRa technology seems to be usable also in indoors applications as measured via packet error rates. In addition, the authors reported the measured power consumptions for the used LoRa transceivers using various communications parameters.

Finally, Editors of this Special Issue would like to thank all the authors for their valuable contribution and collaboration. We would also like to thank Prof. Kaveh Pahlavan, Editor-in-Chief, for the possibility to make this Special Issue.

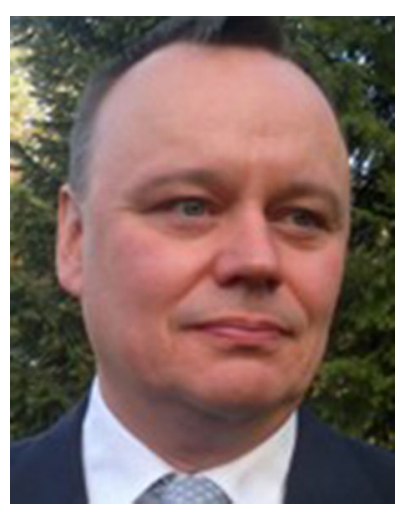

Dr. Matti Hämäläinen received his M.Sc., Lic.Tech., and Dr.Sc. degrees in 1994, 2002 and 2006, respectively, all from the University of Oulu, Finland. He has more than 160 international scientific publications and one patent. He is coauthor of "Wireless UWB Body Area Networks: Using the IEEE802.15.4-2011" by Academic Press, and co-editor of "UWB: Theory and Applications" by Wiley. His current position is University Researcher and Adjunct Professor at Centre for Wireless Communications at University of Oulu, and IAS Visiting Professor at Yokohama National University, Japan. His research interests include UWB systems, wireless body area networks, medical ICT, and radio channel modeling.

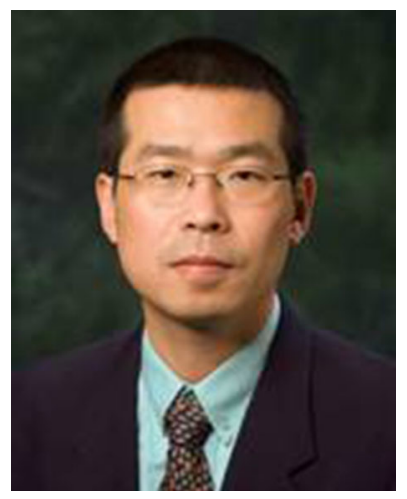

Dr. Xinrong Li received B.E. degree from the University of Science and Technology of China, Hefei, China, in 1995, M.E. degree from the National University of Singapore in 1999, and Ph.D. degree from Worcester Polytechnic Institute (WPI), Worcester, MA, in 2003, all in Electrical Engineering. From 2003 to 2004, he was a Postdoc Research Fellow at the Center for Wireless Information Network Studies, WPI. He joined the Department of Electrical Engineering, University of North Texas,

Denton, Texas, as an Assistant Professor in 2004, and then he became an Associate Professor in 2010. His recent research has been focused on statistical signal processing, real-time embedded system design, wireless sensor networks, and Internet of Things, among others. 